\title{
CLASIFICACIÓN DE SUELOS DE LA ZONA NORESTE Y NOROESTE DE SAN PEDRO SULA
}

\author{
Melvin Joel Morales Fuentes ${ }^{1}$, Leda Mercedes Gale Torre ${ }^{2}$ \\ y Raúl Edgardo Fernández Hernández \\ Estudiantes de la Carrera de Ingeniería Civil de la Universidad Tecnológica Centroamericana \\ (UNITEC), Campus San Pedro Sula ${ }^{3}$
}

(Recibido: Julio, 2016/ Aceptado: Julio, 2017)

\begin{abstract}
Resumen
La investigación tiene como principal objetivo clasificar el suelo del sector Noreste y Noroeste de la ciudad de San Pedro Sula por medio del Método SUCS (Sistema Unificado de Clasificación de Suelos). Se logró dividiendo dichos sectores en nueve cuadrantes, para formar un área total de $9 \mathrm{~km} 2$ y así tomar muestras, a cada $0.50 \mathrm{~m}$, de suelo hasta llegar a $2 \mathrm{~m}$ de profundidad. Se realizaron 92 pruebas de granulometría (ASTM C -136), gravedad específica (ASTM D-558) e hidrometrías (ASTM D-422) para determinar el tipo de suelo que existe por estratos y validar los datos utilizando métodos estadísticos. La hipótesis de investigación planteada es que, en la zona de estudio, según la clasificación SUCS, al menos el 45\% del suelo es de tipo SM (arena limosa). En función de ésta se concluyó que las clasificaciones de los suelos presentes en la zona de investigación son: arena mal graduada con limos (SP-SM), arena bien graduada con limos (SW-SM), arena limosa (SM), arena mal graduada (SP), arena bien graduada (SW), grava mal graduada (GP), grava bien graduada (GW), grava limosa (GM). Siendo el tipo arena limosa (SM) el más predominante, obteniendo un $32.61 \%$ del total de las muestras.
\end{abstract}

Palabras Claves: Suelos, Método SUCS, granulometría, gravedad específica, hidrometrías

\begin{abstract}
The main objective of the investigation is to classify the soil of the Northeast and Northwest sector of the city of San Pedro Sula through the USCS (Unified Soil Classification System) Method. It was achieved by dividing these sectors into nine quadrants, to form a total area of 9 $\mathrm{km} 2$ and to take samples, every $0.50 \mathrm{~m}$, of soil to $2 \mathrm{~m}$ depth. There were 92 tests of granulometry (ASTM C -136), specific gravity (ASTM D-558) and hydrometrics (ASTM D422) to determine the type of soil that exists by strata and validate the data using statistical methods. The research hypothesis was that in the study area according to the USCS classification of at least $45 \%$ of the soil is type SM (silty sand). It was concluded that the classification of the soils present in the research area are: poorly graded sand (SW-SM), silty sand (SM), poorly graded sand (SP), well graded sand (SW), poorly graded gravel (GP), well
\end{abstract}

\footnotetext{
${ }^{1}$ Autor para correspondencia. Email melvinmorales0994@gmail.com

2 Autor para correspondencia. Email ledagale@gmail.com

${ }^{3}$ Michael Job Pineda Canales fungió como Asesor Temático y Mario Humberto Cárdenas Murillo como Asesor Metodológico
} 
graded gravel (SP-SM) GW), records slime (GM). The sand silt type (SM) is the most predominant, obtaining $32.61 \%$ of the total samples.

Keywords: Soil, USCS method, granulometry, specific gravity, hydrometric

\section{Introducción}

El éxito de una obra depende del cuidado en cada uno de los detalles que pueden influir en ella. El estudio de suelos es de vital importancia en el desarrollo de un proyecto. El conocimiento del material con el que se cuenta en el sitio es muy útil para la toma de decisiones.

Estos análisis le proporcionan al proyectista información que debe ser considerada, ya que es un factor determinante en el diseño. El primer paso es la clasificación del material en el proyecto, pues es un indicador del tipo de estudio a realizar, es decir, qué pruebas se deben realizar para conocer las propiedades físicas y mecánicas del terreno.

Cabe resaltar que omitir estudios de esta naturaleza puede dar como resultado distintos daños en las estructuras como fisuras, grietas, inutilización de la obra y en ocasiones hasta el colapso de la misma. No realizar la exploración previa de todas las características de la zona del proyecto, es innecesario, pues los costos de estos son relativamente bajos, en comparación a los que se podrían producir por los daños causados en las estructuras.

La presente investigación consiste en dividir la ciudad en cuadrantes de 1x1 km y llevar a cabo la clasificación de los suelos presentes, con el objetivo de en el futuro diseñar un mapa que represente los tipos de suelo en la ciudad de San Pedro Sula.

La investigación abarca la zona Noroeste y Noreste de la ciudad de San Pedro Sula, las cuales abarcan los siguientes barrios y colonias: Villas del Sol, Bermejo, Jardines del Valle, Stibys, Los Médicos, Buenos Aires, El Roble, Los Laureles, Colonia Universidad, Rancho El Coco, Guadalupe, La Esperanza, Bográn, La Colombia, Santa Ana, Las Flores, Zerón, San Cristóbal y Valle Azul.

\section{Metodología}

Los enfoques de una investigación pueden ser: enfoque cuantitativo y el cualitativo. De acuerdo a Hernández, Fernández y Baptista (2010), el enfoque cualitativo es aquel que "...utiliza la recolección de datos sin medición numérica para descubrir o afinar preguntas de investigación en el proceso de interpretación" (p.7). por su parte, el enfoque cuantitativo, es aquel que "...usa la recolección de datos para probar hipótesis, con base en la medición numérica y el análisis estadístico, para establecer patrones de comportamiento y probar teorías" (Hernández, Fernández \& Baptista, 2010, p.4).

Para la presente investigación el enfoque utilizado es principalmente el cuantitativo, debido a que, para llegar a establecer conclusiones, es necesario llevar a cabo una serie de pruebas con datos numéricos. 
A continuación, en la Figura 1 se presenta un esquema que explica la manera de proceder en el enfoque cuantitativo. Seguir el orden establecido por este proceso es muy útil, pues ayuda a estructurar de mejor manera la investigación.

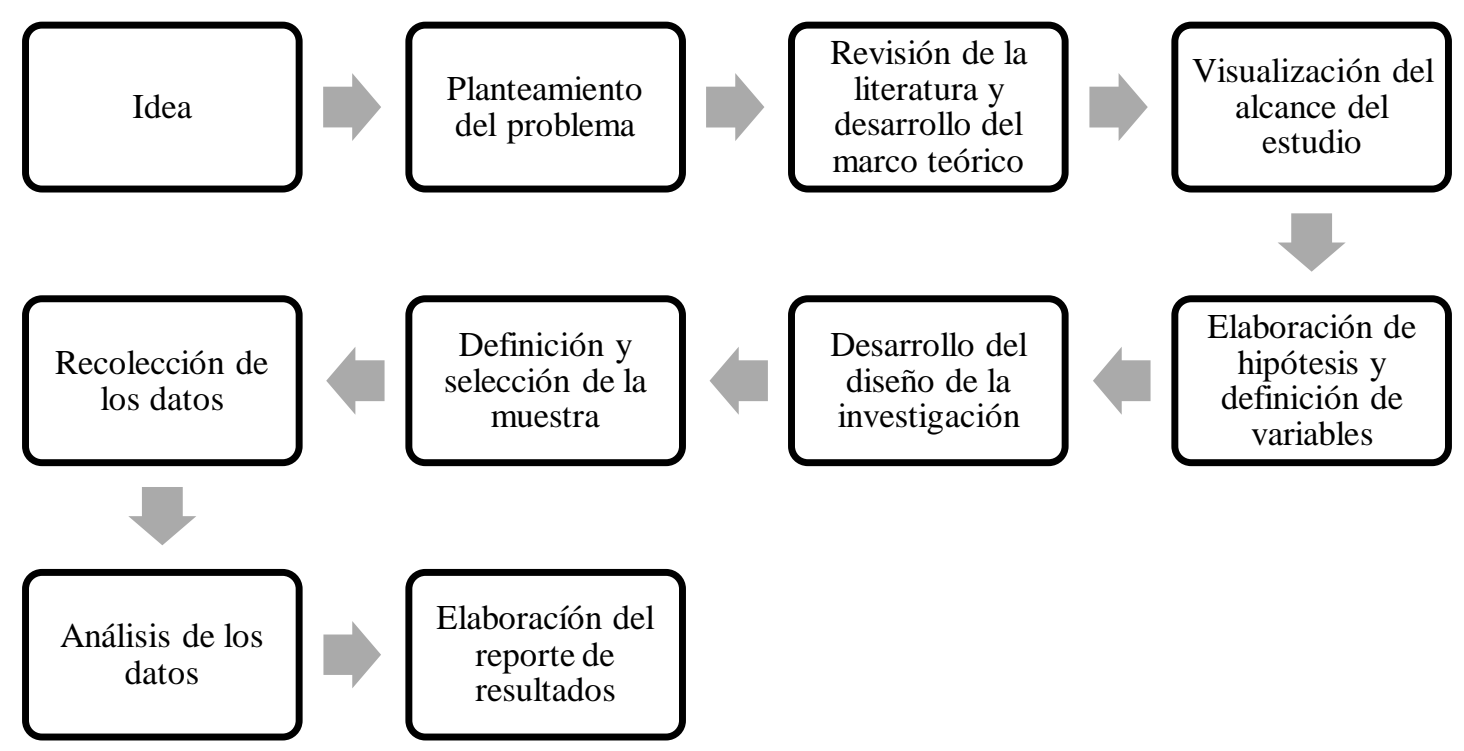

Figura 1: Proceso Cuantitativo

Fuente: Hernández Sampieri, R., Fernández Collado, C., \& Baptista Lucio, P. (2010). Metodología de la Investigación (5. ${ }^{a}$ ed.).

La Figura 1 muestra cómo llevar un orden específico permite o facilita el curso de la investigación, pues de esta manera se sabe la forma en la que se debe proceder sin perder de vista ningún elemento del proyecto.

Agregado a esto, se debe señalar que la presente investigación es del tipo no experimental pues no se tiene control sobre las variables independientes, es decir, estos no se pueden manipular. El trabajo consiste básicamente en someter el material a pruebas de laboratorio para poder llevar a cabo una clasificación del mismo. La investigación no experimental es también conocida como investigación Ex Post Facto, término que proviene del latín y significa después de ocurridos los hechos. De acuerdo con Kerlinger (1983) la investigación Ex Post Facto es un tipo de “... investigación sistemática en la que el investigador no tiene control sobre las variables independientes porque ya ocurrieron los hechos o porque son intrínsecamente manipulables," (p. 269). En la investigación Ex Post Facto los cambios en la variable independiente ya ocurrieron y el investigador tiene que limitarse a la observación de situaciones ya existentes dada la incapacidad de influir sobre las variables y sus efectos (Hernández, Fernández y Baptista, 1991).

La población a estudiar la constituyen nueve cuadrantes que forman un área total de 9 $\mathrm{km}^{2}$. Dentro de los nueve cuadrantes se tomarán 23 calicatas para la toma de muestras y su posterior análisis en el laboratorio. El sector Noroeste y Noreste de la ciudad de San Pedro Sula es la zona que abarca la investigación. Los especímenes serán extraídos a cada 0.50 metros para alcanzar un total de 2.00 metros, donde el suelo se encuentra prácticamente inalterado. 
La elección de las calicatas se escogió con la ayuda de un mapa de San Pedro Sula en archivo AutoCAD. Sobre él se dibujó un rectángulo de $9 \mathrm{~km}^{2}, 3 \mathrm{~km}$ de ancho por $3 \mathrm{~km}$ de alto, que abarcó la zona Noroeste y Noreste. Luego, el rectángulo se dividió en 9 cuadrantes, de $1 \mathrm{~km}^{2}$ cada uno. Una vez realizada su división, se tomó la decisión de extraer muestras en las esquinas y en el centro de cada cuadrante, de manera que se obtuvieran 23 puntos de extracción. Finalmente, con la ayuda de Google Maps, se marcó el lugar exacto para su visita y se procedió con los permisos de los propietarios para la realización de la calicata.

Las muestras de suelo se obtuvieron con la excavación de calicatas con dimensiones de 1.40 x 0.60 x 2.00 metros. Se contó con la ayuda de dos jornaleros para su extracción. Una vez que se obtuvieron los permisos para cavar, se procedió al sitio y se seleccionó un punto donde no se apreciara ninguna conexión de agua potable o cualquier tipo de obstáculo importante; se comenzó la excavación y se tomaron muestras a cada 0.50 metros hasta alcanzar los 2.00 metros de profundidad. Las muestras fueron luego embolsadas y rotuladas.

Tabla 1: Matriz metodológica.

\begin{tabular}{|c|c|c|c|c|c|c|}
\hline \multirow[t]{2}{*}{ Título } & \multirow[t]{2}{*}{ Problema } & \multirow{2}{*}{$\begin{array}{l}\text { Preguntas de } \\
\text { Investigación }\end{array}$} & \multicolumn{2}{|c|}{ Objetivos } & \multicolumn{2}{|c|}{ Variables } \\
\hline & & & General & Específicos & Independientes & Dependiente \\
\hline \multirow{3}{*}{$\begin{array}{l}\text { Clasificación } \\
\text { de suelos de la } \\
\text { zona noreste y } \\
\text { noroeste de } \\
\text { San Pedro Sula }\end{array}$} & \multirow{3}{*}{$\begin{array}{l}\text { ¿Cuáles } \\
\text { son los } \\
\text { tipos de } \\
\text { suelos } \\
\text { presentes } \\
\text { en las } \\
\text { distintas } \\
\text { zonas de la } \\
\text { ciudad de } \\
\text { San Pedro } \\
\text { Sula? }\end{array}$} & $\begin{array}{c}\text { ¿Qué } \\
\text { información es } \\
\text { necesario } \\
\text { obtener de las } \\
\text { pruebas de } \\
\text { laboratorio } \\
\text { para llevar a } \\
\text { cabo la } \\
\text { clasificación de } \\
\text { un suelo? }\end{array}$ & \multirow{3}{*}{$\begin{array}{c}\text { Identificar los } \\
\text { diferentes tipos } \\
\text { de suelos } \\
\text { presentes en la } \\
\text { zona de } \\
\text { investigación. }\end{array}$} & $\begin{array}{c}\text { Obtener la } \\
\text { información de } \\
\text { las pruebas de } \\
\text { laboratorio que } \\
\text { es relevante para } \\
\text { la } \\
\text { caracterización } \\
\text { de los suelos. }\end{array}$ & $\begin{array}{l}\text { Pruebas de } \\
\text { muestreo de } \\
\text { laboratorio }\end{array}$ & \multirow{3}{*}{$\begin{array}{c}\text { Caracterización } \\
\text { de los suelos en } \\
\text { la zona noreste y } \\
\text { noroeste de la } \\
\text { ciudad. }\end{array}$} \\
\hline & & $\begin{array}{l}\text { ¿Qué variación } \\
\text { existe en el } \\
\text { tipo de suelo } \\
\text { presente según } \\
\text { la profundidad } \\
\text { de la muestra? }\end{array}$ & & $\begin{array}{l}\text { Identificar los } \\
\text { tipos de suelo } \\
\text { que existen a las } \\
\text { distintas } \\
\text { profundidades. }\end{array}$ & Perfil del suelo & \\
\hline & & $\begin{array}{l}\text { ¿Qué relación } \\
\text { existe en el } \\
\text { suelo de los } \\
\text { diferentes } \\
\text { puntos a } \\
\text { estudiar? }\end{array}$ & & $\begin{array}{c}\text { Reconocer los } \\
\text { tipos de suelos } \\
\text { en los distintos } \\
\text { puntos de } \\
\text { estudio. }\end{array}$ & $\begin{array}{l}\text { Ubicación de los } \\
\text { puntos estudio. }\end{array}$ & \\
\hline
\end{tabular}

Fuente: Elaboración Propia

En la Tabla 1 se muestra la matriz metodológica utilizada en el proyecto, la cual es de gran utilidad para verificar si hay concordancia o congruencia en las distintas partes de este. El desarrollar este tipo de trabajos, permite al investigador tener una idea clara de cuál es el enfoque que se debe seguir. 
En la Tabla 1 se observa como existe una relación directa entre las preguntas de investigación y los objetivos, así como también con las variables, con esto se puede estar seguro que lo que se espera obtener al final del trabajo va a ser logrado. Por ser éste un estudio exploratorio, se prescinde de una hipótesis de investigación.

En función de lo anterior, para el desarrollo de la investigación, se utilizan los siguientes métodos:

- Análisis granulométrico por tamizado (ASTM D 422)

La granulometría es uno de los métodos más antiguos y comunes, pues brinda información básica para determinar la uniformidad o graduación de un material dentro de rangos ya establecidos, que permiten determinar la clasificación de este suelo.

- Gravedad específica de los suelos (ASTM D 854).

La gravedad específica es la relación del peso de una muestra en el aire, de un volumen que ya se haya calculado o que ya se conozca, contra el peso en el aire de una misma cantidad de volumen de agua destilada a una temperatura de cuatro Celsius.

- Análisis granulométrico por hidrómetro (ASTM D 422)

El ensayo por hidrómetro se basa en la sedimentación de un material en suspensión en un líquido; sirve para la determinación de la variación de la densidad de la suspensión con el transcurso del tiempo y medir la altura de caída del gramo de tamaño más grande correspondiente a la densidad media.

Para complementar lo anterior, es necesario señalar que Hernández, Fernández\& Baptista Lucio (2010) comentan que,"...el análisis cuantitativo de los datos se lleva a cabo por computadora u ordenador. El análisis de los datos se efectúa sobre la matriz de datos utilizando un programa computacional"(p. 278). A continuación, se esquematiza dicho proceso.

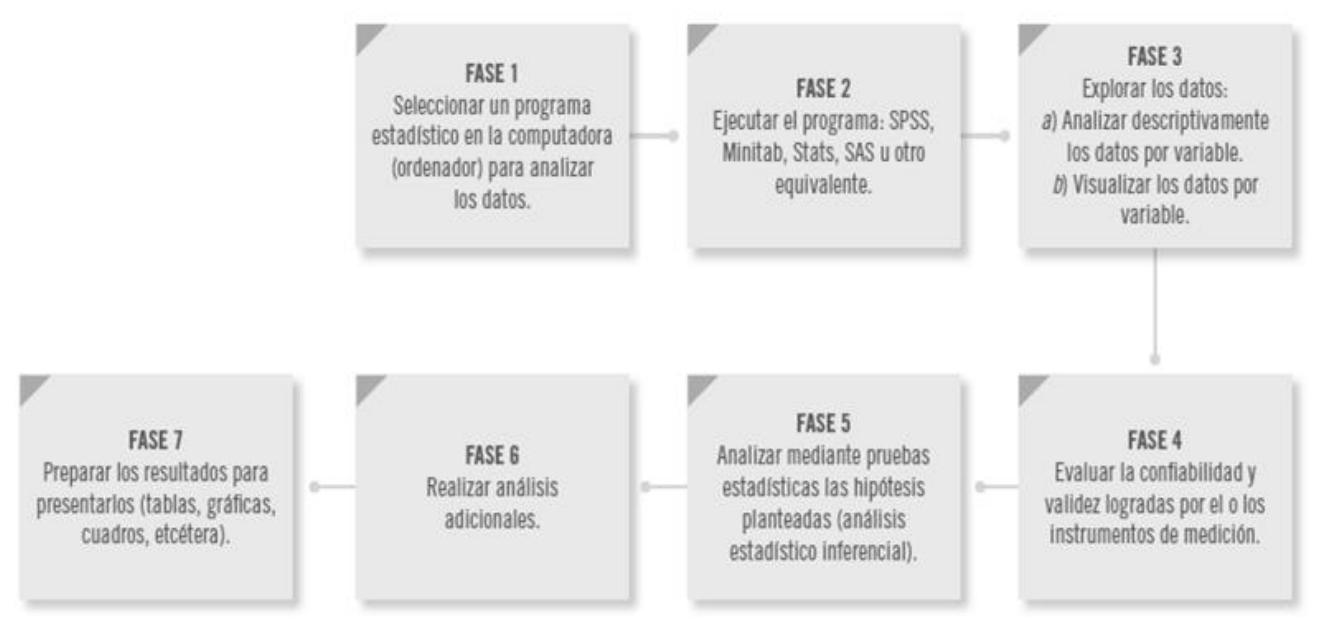

Figura 2. Proceso para efectuar análisis estadístico.

Fuente: Bernal, C. A. (2010). Metodología de la Investigación (3. ${ }^{\mathrm{a}}$ ed.). Colombia: Pearson Educación

En la Figura 2 se ilustra el proceso para realizar un análisis de forma cuantitativa de los datos. 
Las pruebas estadísticas utilizadas en el proceso de ésta investigación son el Método de las Proporciones, método ANOVA, y la Prueba T.

- Método de las proporciones

Las pruebas de proporciones son adecuadas cuando, los datos que se están analizando, constan de cuentas o frecuencias de elementos de dos o más clases. El objetivo de estas pruebas es evaluar las afirmaciones con respecto a una proporción de población. Las pruebas se basan en la premisa de que una proporción muestral será igual a la proporción verdadera de la población si se toman márgenes o tolerancias para la variabilidad muestral. Las pruebas suelen enfocarse en la diferencia entre un número esperado de ocurrencias, suponiendo que una afirmación es verdadera, y el número observado realmente. La diferencia se compara con la variabilidad prescrita mediante una distribución de muestreo que tiene como base el supuesto de que Ho es realmente verdadera.

\section{- Método ANOVA}

Un análisis de varianza (ANOVA) prueba la hipótesis de que las medias de dos o más poblaciones son iguales. Los ANOVA evalúan la importancia de uno o más factores al comparar las medias de la variable de respuesta en los diferentes niveles de los factores. La hipótesis nula establece que todas las medias de la población (medias de los niveles de los factores) son iguales mientras que la hipótesis alternativa establece que al menos una es diferente.

\section{- Prueba T}

La prueba t pareada calcula la diferencia dentro de cada par de mediciones de una muestra antes y después de un acontecimiento o un cambio, determina la media de dichas modificaciones e informa si la media de las diferencias es estadísticamente significativa.

En la Tabla 2 se muestra lo necesario para llevar a cabo las pruebas de laboratorio de granulometría, gravedad específica e hidrometría.

Tabla 2: Equipo y material requerido según prueba de laboratorio.

\begin{tabular}{|c|c|c|c|c|c|}
\hline \multicolumn{2}{|c|}{ Granulometría } & \multicolumn{2}{|c|}{ Gravedad Específica } & \multicolumn{2}{|l|}{ Hidrometría } \\
\hline Equipo & Material & Equipo & Material & Equipo & Material \\
\hline $\begin{array}{l}\text { Balanzas } \\
\text { Tamices } \\
\text { Horno } \\
\text { Charolas } \\
\text { Cepillo Metálico } \\
\text { Mezcladora mecánica }\end{array}$ & Suelo $(1,000 \mathrm{grs})$ & \begin{tabular}{|l|} 
Picnómetro \\
Estufa de gas \\
Balanza \\
Horno \\
Termómetro \\
Tara \\
Embudo \\
Martillo de goma \\
Placa de asbesto \\
Cronómetro
\end{tabular} & $\begin{array}{c}\text { Suelo y } \\
\text { Agua } \\
\text { destilada }\end{array}$ & $\begin{array}{l}\text { Balanza } \\
\text { Probeta de 1,000 ml } \\
\text { Cilindro de vidrio } \\
\text { Termómetro } \\
\text { Beaker } \\
\text { Mezcladora mecánica } \\
\text { Cronómetro } \\
\text { Frasco lavador } \\
\text { Hidrómetro ASTM } 152 \mathrm{H}\end{array}$ & $\begin{array}{l}\text { Suelo,Agua } \\
\text { destilada y } \\
\text { Agente } \\
\text { dispersante }\end{array}$ \\
\hline
\end{tabular}

Fuente: Normas ASTM C -136, ASTM D-558 y ASTM D-422

Estos instrumentos y materiales fueron basados en las normas ASTM. 


\begin{tabular}{|c|c|c|c|c|c|}
\hline Tamiz no. & $\begin{array}{c}\text { Tamaño Tamiz } \\
(\mathrm{mm})\end{array}$ & $\begin{array}{l}\text { Peso Retenido } \\
\text { Parcial en gramos }\end{array}$ & $\begin{array}{l}\% \text { Retenido } \\
\text { Parcial }\end{array}$ & $\begin{array}{l}\text { \% Retenido } \\
\text { Acumulado }\end{array}$ & $\begin{array}{l}\text { \% Que pasa } \\
\text { por el tamiz }\end{array}$ \\
\hline $11 / 2$ & 38.1 & & & & \\
\hline 1 & 25 & & & & \\
\hline $3 / 4$ & 19 & & & & \\
\hline $1 / 2$ & 12.5 & & & & \\
\hline $3 / 8$ & 9.5 & & & & \\
\hline No. 4 & 4.75 & & & & \\
\hline NNo. 10 & 2 & & & & \\
\hline No. 40 & 0.425 & - & & & \\
\hline No. 200 & 0.075 & 40 & & & \\
\hline \multirow[t]{2}{*}{ Fondo } & - & & & & \\
\hline & Suma & 0.00 & 0.00 & & \\
\hline \multirow[t]{2}{*}{$\mathrm{D}_{10}(\mathrm{~mm}):$} & & \multirow[t]{2}{*}{$\mathrm{D}_{30}(\mathrm{~mm}):$} & & \multirow[t]{2}{*}{$\mathrm{D}_{60}(\mathrm{~mm}):$} & \\
\hline & Cu: & & $\mathrm{Cz}$ & & \\
\hline ए\% & 0.00 & & & & \\
\hline LP\% & 0.00 & Plasticidad & & & \\
\hline IP\% & 0.00 & $\mathbf{X}$ & & & \\
\hline
\end{tabular}

Figura 3: Proceso para efectuar análisis estadístico.

Fuente: Elaboración Propia

En la Figura 3 se muestra el formato utilizado para realizar la clasificación de suelos de las distintas muestras tomadas.

\section{Resultados}

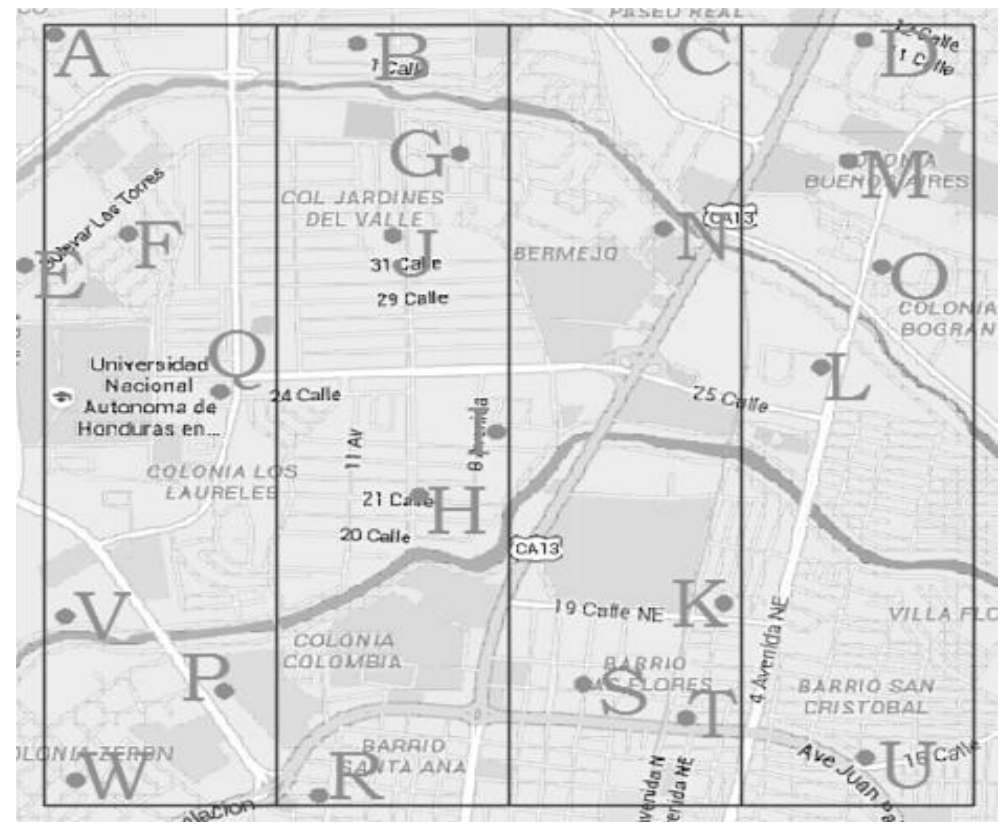

Figura 4: Mapa para Análisis Estadístico.

Fuente: Elaboración Propia 
En la Figura 4 se muestra la división de los cuadrantes para el análisis estadístico ANOVA. Se dividió en 4 partes de manera que cada cuadrante quedara con las mismas cantidades de muestras posibles y que a la hora de su análisis, los datos se organizaran de manera uniforme. Las variables a comparar fueron la gravedad específica, el porcentaje de suelo que pasa el tamiz \#200, el coeficiente de curvatura y finalmente el coeficiente de uniformidad.

En la Tabla 3 se muestran los resultados obtenido para las comparaciones de datos en los distintos sectores y profundidades. En la tabla 3 se encuentran los resultados que fueron obtenidos por medio del programa Minitab, se presentan los valores resultantes, los cuales son necesarios para hacer las respectivas conclusiones en cada uno de los casos.

Tabla 3: Tabla resumen de resultado Método ANOVA.

\begin{tabular}{|l|c|c|c|c|}
\hline \multicolumn{4}{|c|}{ Comparaciones } & \multicolumn{2}{|c|}{ Sector } & \multicolumn{2}{c|}{ Profundidad } \\
\cline { 2 - 5 } & Valor & Conclusión & Valor & Conclusión \\
\hline Gravedad Específica & 0.258 & No difiere & 0.014 & Difiere \\
\hline \% Pasa tamiz \#200 & 0.002 & Difiere & 0.792 & No difiere \\
\hline Coeficiente de uniformidad & 0.187 & No difiere & 0.169 & No difiere \\
\hline Coeficiente de curvatura & 0.011 & Difiere & 0.562 & No difiere \\
\hline
\end{tabular}

Fuente: Elaboración Propia

- $\quad$ Mapas de la zona estudiada

En la Figura 5 se muestra la simbología que se utilizó en la elaboración de los mapas de zonificación en cada una de las profundidades de estudio.

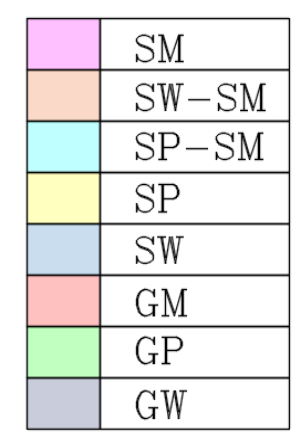

Figura 5. Simbología utilizada en la elaboración de los mapas de zonificación.

Fuente: Elaboración Propia 
La Figura 5 permite identificar qué tipo de suelo existe en cada una de las zonas del cuadrante de estudio. La figura 6 muestra la zona de estudio con sus distintos tipos de suelos a 0.50 metros de profundidad.

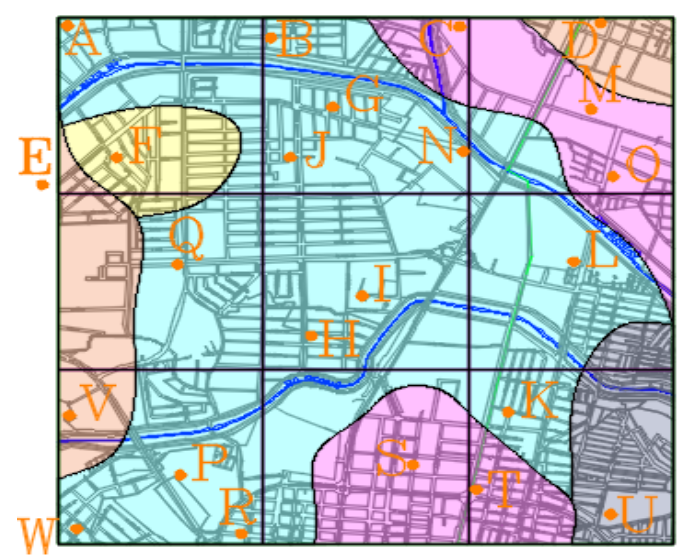

Figura 6: Mapa de tipos de suelos a 0.50 metros

Fuente: Elaboración Propia

En la Figura 6 se observa que los tipos de suelos existentes son arena mal graduada con limos (SP-SM), arena bien graduada con limos (SW-SM), arena limosa (SM), arena mal graduada (SP) y arena bien graduada (SW). Siendo el más prominente, arena mal graduada con limos (SP-SM) con un porcentaje de $56.52 \%$.

La Figura 7 muestra la zona de estudio con sus distintos tipos de suelos a 1.00 metros de profundidad.

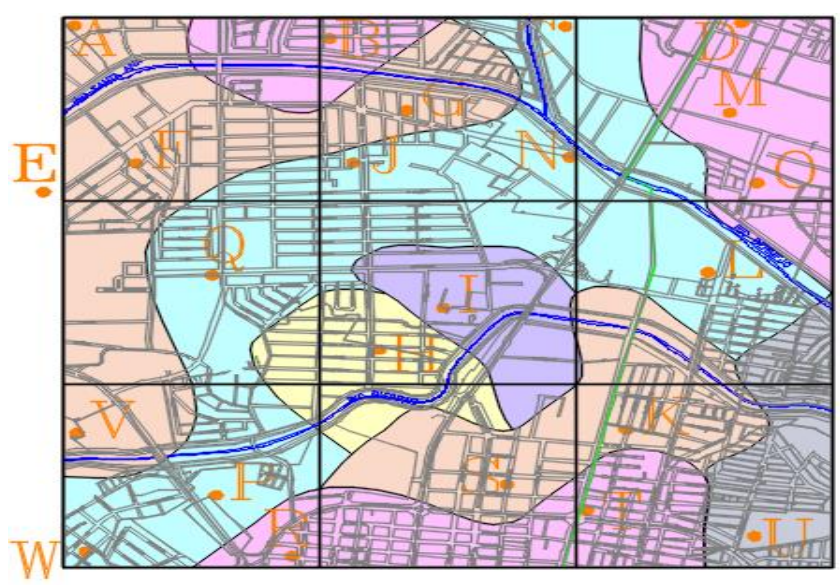

Figura 7: Mapa de tipos de suelos a 1.00 metros

Fuente: Elaboración Propia

En la Figura 7 se observa que los tipos de suelos existentes son arena mal graduada con limos (SP-SM), arena bien graduada con limos (SW-SM), arena limosa (SM), arena mal graduada (SP), arena bien graduada (SW) y grava bien graduada $(\mathrm{GW})$. Siendo el 
más prominente, arena mal graduada con limos (SP-SM), arena bien graduada con limos (SW-SM) y arena limosa (SM) con un porcentaje de $30.43 \%$.

La Figura 7 muestra la zona de estudio con sus distintos tipos de suelos a 1.50 metros de profundidad.

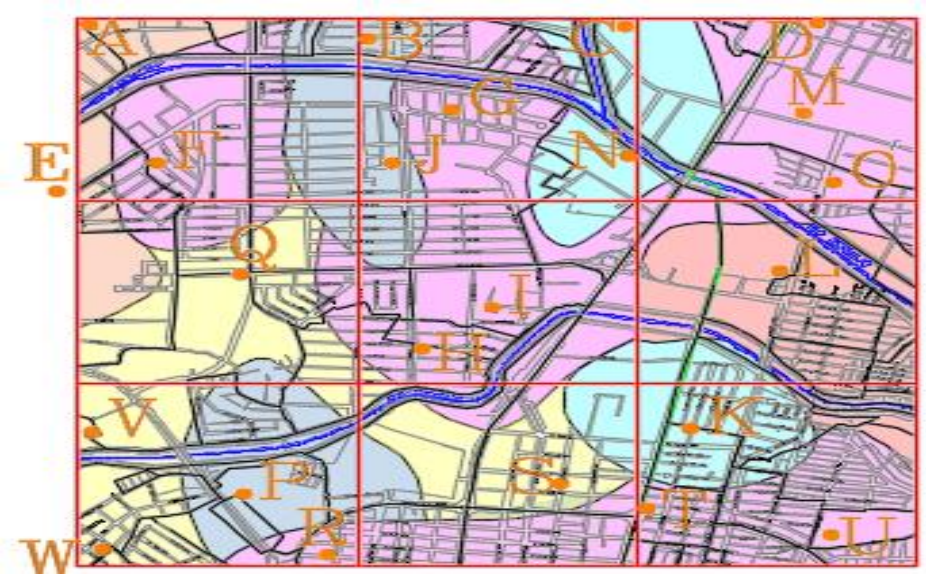

Figura 8: Mapa de tipos de suelos a 1.50 metros

Fuente: Elaboración Propia

En la Figura 8 se observa que los tipos de suelos existentes son arena mal graduada con limos (SP-SM), arena bien graduada con limos (SW-SM), arena limosa (SM), arena mal graduada (SP), arena bien graduada (SW) y grava limosa (GM). Siendo el más prominente, arena limosa (SM) con un porcentaje de $34.78 \%$.

La Figura 9 muestra la zona de estudio con sus distintos tipos de suelos a 2.00 metros de profundidad.

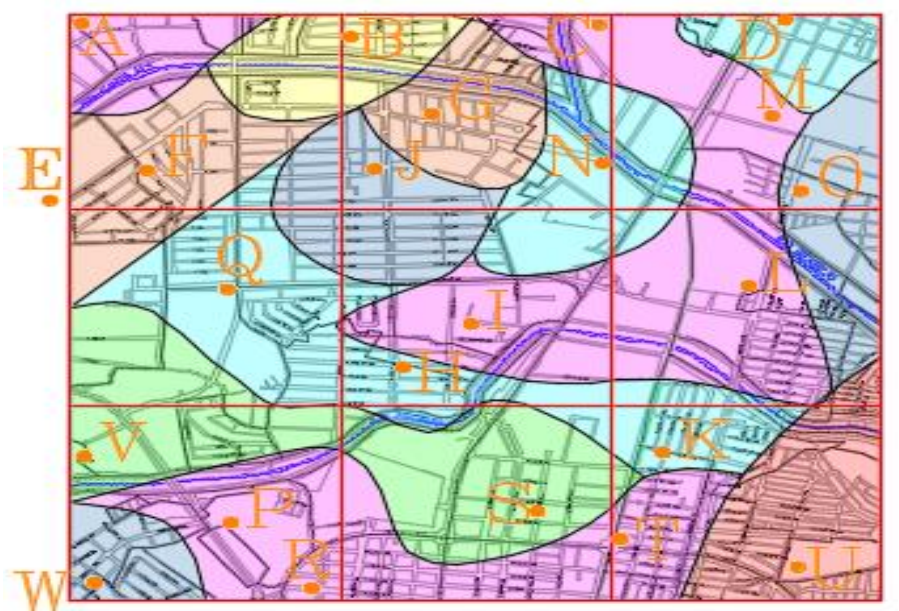

Figura 9: Mapa de tipos de suelos a 2.00 metros

Fuente: Elaboración Propia

En la Figura 9 se observa que los tipos de suelos existentes son arena mal graduada con limos (SP-SM), arena bien graduada con limos (SW-SM), arena limosa (SM), arena 
mal graduada (SP), arena bien graduada (SW), grava mal graduada (GP) y grava limosa (GM). Siendo el más prominente, arena limosa (SM) con un porcentaje de $43.48 \%$.

En la Figura 10 se muestra la clasificación de suelos general con los diferentes tipos de suelos por profundidad y el número de ejemplares encontrados.

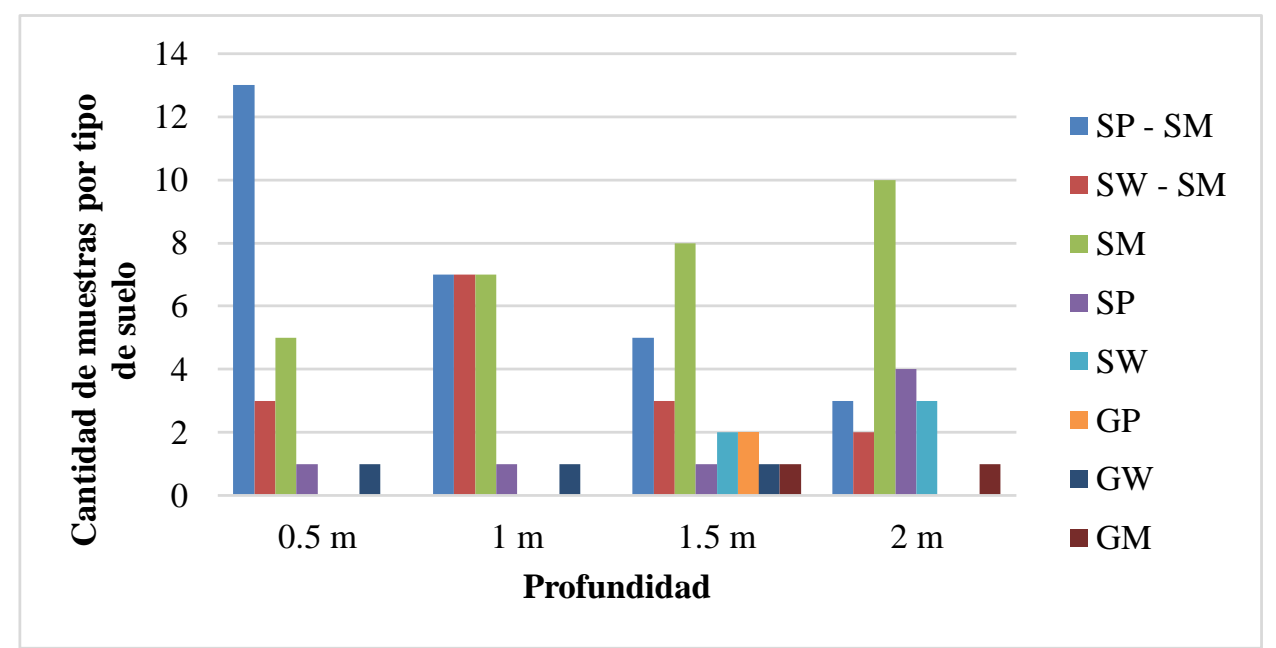

Figura 10. Clasificación de Suelos Según su Profundidad

Fuente: Elaboración Propia

La Tabla 4, muestra los resultados de los ensayos de Gravedad específica para cada una de las muestras, especificando la profundidad de la misma

Tabla 4: Clasificación de suelos según su profundidad.

\begin{tabular}{|c|c|c|c|c|c|c|c|c|}
\hline \multicolumn{9}{|c|}{ Tabla Resumen de Gravedades Especificas } \\
\hline Muestra & $\begin{array}{l}\text { Profundidad } \\
\text { (m) }\end{array}$ & (Gs) & Muestra & $\begin{array}{l}\text { Profundidad } \\
\text { (m) }\end{array}$ & (Gs) & Muestra & $\begin{array}{c}\text { Profundidad } \\
\text { (m) }\end{array}$ & (Gs) \\
\hline Al & 0.50 & 2.37 & H4 & 2.00 & 2.47 & P3 & 1.50 & 2.31 \\
\hline A2 & 1.00 & 2.15 & Il & 0.50 & 2.29 & P4 & 2.00 & 2.41 \\
\hline$A^{3}$ & 1.50 & 2.41 & I2 & 1.00 & 2.46 & Q1 & 0.50 & 2.32 \\
\hline A4 & 2.00 & 2.71 & I3 & 1.50 & 2.68 & Q2 & 1.00 & 2.42 \\
\hline B1 & 0.50 & 2.44 & 14 & 2.00 & 2.71 & Q3 & 1.50 & 2.35 \\
\hline B2 & 1.00 & 2.37 & J1 & 0.50 & 2.38 & Q4 & 2.00 & 2.33 \\
\hline B3 & 1.50 & 2.54 & J2 & 1.00 & 2.36 & R1 & 0.50 & 2.36 \\
\hline B4 & 2.00 & 2.45 & J3 & 1.50 & 2.38 & R2 & 1.00 & 2.47 \\
\hline C1 & 0.50 & 2.41 & J4 & 2.00 & 2.35 & R3 & 1.50 & 2.37 \\
\hline $\mathrm{C}_{2}$ & 1.00 & 2.37 & KI & 0.50 & 2.37 & R4 & 2.00 & 2.50 \\
\hline $\mathrm{C}_{3}$ & 1.50 & 2.44 & K2 & 1.00 & 2.14 & S1 & 0.50 & 2.44 \\
\hline C4 & 2.00 & 2.43 & K3 & 1.50 & 2.34 & S2 & 1.00 & 2.40 \\
\hline D1 & 0.50 & 2.29 & K4 & 2.00 & 2.35 & S3 & 1.50 & 2.49 \\
\hline D2 & 1.00 & 2.20 & L1 & 0.50 & 2.38 & S4 & 2.00 & 2.39 \\
\hline D3 & 1.50 & 2.32 & L2 & 1.00 & 2.31 & T1 & 0.50 & 2.57 \\
\hline D4 & 2.00 & 2.35 & L3 & 1.50 & 2.37 & $\mathrm{~T} 2$ & 1.00 & 2.54 \\
\hline E1 & 0.50 & 2.34 & L4 & 2.00 & 2.50 & T3 & 1.50 & 2.84 \\
\hline E2 & 1.00 & 2.33 & MI & 0.50 & 2.40 & T4 & 2.00 & 2.50 \\
\hline E3 & 1.50 & 2.45 & M2 & 1.00 & 2.36 & U1 & 0.50 & 2.54 \\
\hline E4 & 2.00 & 2.33 & M3 & 1.50 & 2.31 & $\mathrm{U} 2$ & 1.00 & 2.46 \\
\hline F1 & 0.50 & 2.37 & M4 & 2.00 & 2.32 & $\mathrm{U} 3$ & 1.50 & 2.46 \\
\hline F2 & 1.00 & 2.39 & N1 & 0.50 & 2.33 & $\mathrm{U} 4$ & 2.00 & 2.45 \\
\hline F3 & 1.50 & 2.42 & N2 & 1.00 & 2.33 & v1 & 0.50 & 2.39 \\
\hline F4 & 2.00 & 2.40 & N3 & 1.50 & 2.34 & V2 & 1.00 & 2.42 \\
\hline G1 & 0.50 & 2.41 & N4 & 2.00 & 2.38 & V3 & 1.50 & 2.56 \\
\hline G2 & 1.00 & 2.29 & 01 & 0.50 & 2.36 & V4 & 2.00 & 2.50 \\
\hline G3 & 1.50 & 2.40 & 02 & 1.00 & 2.38 & w1 & 0.50 & 2.59 \\
\hline $\mathrm{G}_{4}$ & 2.00 & 2.30 & 03 & 1.50 & 2.61 & w2 & 1.00 & 2.24 \\
\hline $\mathrm{Hl}$ & 0.50 & 2.44 & O4 & 2.00 & 2.32 & wh3 & 1.50 & 2.57 \\
\hline H2 & 1.00 & 2.39 & P1 & 0.50 & 2.43 & w4 & 2.00 & 2.23 \\
\hline $\mathrm{H}^{3}$ & 1.50 & 2.92 & P2 & 1.00 & 2.39 & & & \\
\hline
\end{tabular}

Fuente: Elaboración Propia 
A partir de los datos encontrados en la tabla 1, se pueden utilizar para completar la curva granulométrica mediante la hidrometría.

Con los datos tabulados para cada una de las muestras, se facilita el cálculo de la gravedad específica promedio del suelo en el área de estudio que es de Gs= $2.415 \mathrm{~g} / \mathrm{cm}^{3}$.

En la Figura 10 se muestra como el valor calculado para $Z=-2.38$ es menor que el valor crítico, $\mathrm{Z}=-1.64$, se rechaza a hipótesis de investigación. Por los datos obtenidos en el estudio, se concluye que con un nivel de confiabilidad de $95 \%$ menos del $45 \%$ del suelo de la zona de estudio es del tipo SM.

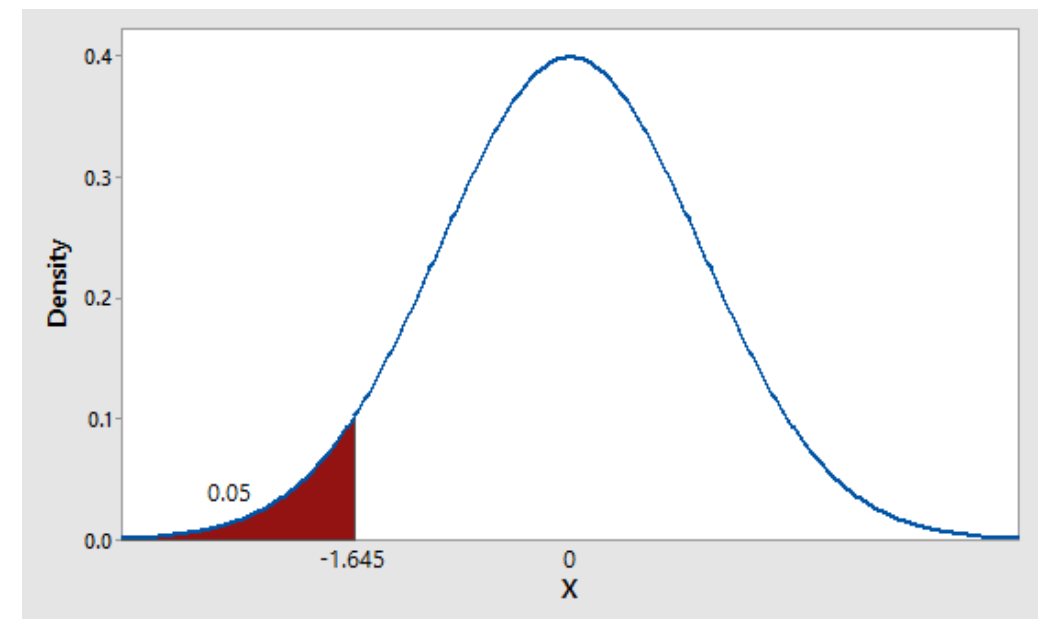

Figura 11: Clasificación de Suelos Según su Profundidad.

Fuente: Walpole, R. E., Myers, R. H., Myers, S. L., \& Ye, K. (2007). Probabilidad y Estadísticas Para Ciencias E Ingenierías (8. ${ }^{a}$ ed.). México: Pearson Educación.

\section{Conclusiones}

Después de la serie de muestras obtenidas, se concluye que:

1. La clasificación de los suelos presentes en la zona de investigación es: arena mal graduada con limos (SP-SM), arena bien graduada con limos (SW-SM), arena limosa $(\mathrm{SM})$, arena mal graduada $(\mathrm{SP})$, arena bien graduada (SW), grava mal graduada (GP), grava bien graduada $(\mathrm{GW})$, grava limosa $(\mathrm{GM})$.

2. Se realizó las pruebas de granulometrías debido a que es el parámetro utilizado para la clasificación por el método SUCS, se hicieron en total 92 de éstos ensayos. Las pruebas de gravedad específica también fueron desarrolladas para cada una de las muestras, esta información fue útil para llevar a cabo análisis de relación entre variables y en los datos necesario para las pruebas de hidrometría. Se determinó que se realizarían 40 de éstos ensayos. No se realizaron las pruebas de límites de Atterberg debido a que el suelo en los sectores de estudios fue del tipo no plástico (NP).

3. Identificación de los tipos de suelos según la profundidad. 
-Profundidad de $0.50 \mathrm{~m}$.

Se encontró que a la profundidad de $0.50 \mathrm{~m}$ el tipo de suelo que predomina es la arena mal graduada con limos (SP-SM) con un porcentaje de $56.52 \%$ y en segundo lugar se encuentra la arena limosa (SM) con un $21.74 \%$.

-Profundidad de $1.00 \mathrm{~m}$.

Se observó que a la profundidad de $1.00 \mathrm{~m}$ existen tres tipos de suelo a igual cantidad arena mal graduada con limos (SP-SM), arena bien graduada con limos (SW-SM) y arena limosa (SM) con un porcentaje de $30.43 \%$.

-Profundidad de $1.50 \mathrm{~m}$.

A la profundidad de $1.50 \mathrm{~m}$ el tipo de suelo que predomina es la arena limosa (SM) con un porcentaje de $34.78 \%$ y en segundo lugar arena mal graduada con limos (SP-SM) con $21.74 \%$.

-Profundidad de $2.00 \mathrm{~m}$.

Se observó que a la profundidad de $2.00 \mathrm{~m}$ el tipo de suelo que existe en mayor cantidad es la arena limosa (SM) con un porcentaje de $43.48 \%$ y en segundo lugar la arena mal graduada (SP) con $17.39 \%$.

4. Con los resultados obtenidos se pudo observar que la proximidad entre cada punto de estudio, no es un indicador de que el suelo en estos sea el mismo, lo cual confirma que antes de hacer cualquier construcción de gran importancia es indispensable realizar los estudios correspondientes.

En referencia a nuestra hipótesis de investigación se concluyó que en la zona de estudios menos del $45 \%$ del suelo es tipo arena limosa (SM), obteniendo un $32.61 \%$ del total de las muestras.

\section{Aplicabilidad}

- Evaluación de la calidad de un suelo como material para subrasante de carreteras.

En la construcción de terraplenes para carreteras, presas de tierra y muchas otras estructuras de ingenierías, los suelos sueltos deben ser compactados para incrementar sus pesos específicos. La compactación incrementa las características de resistencia de los suelos, aumentando así la capacidad de carga de las cimentaciones construidas sobre ellos.

- Determinación de maquinaria y equipo a utilizar.

La mayor parte de las compactaciones de campo se hacen con compactadoras de rodillo los cuales se usan para determinados tipos de suelos:

- Las compactadoras de rodillo lisos son apropiadas para rodadas de pruebas de subrasante y para la operación final de rellenos con suelo arenoso y arcilloso.

- Las compactadoras con neumático de hule son mejores que los de rodillo liso y se utilizan para la compactación de suelos arenosos y arcillosos. 
- Los rodillos pata de cabra son tambores con gran número de turbulencias y tienen su mayor efectividad en la compactación de suelos arcillosos.

- Los rodillos vibratorios son muy eficientes en la compactación de suelos granulares.

- Realización de presupuestos

El proceso de identificar los estratos de depósitos que subyacen a una estructura propuesta y sus características físicas se denomina generalmente exploración del subsuelo, cuyo propósito es obtener información que ayude al ingeniero en geotecnia en las siguientes tareas:

- Seleccionar el tipo y profundidad de una cimentación adecuada para una estructura dada.

- Evaluar la capacidad de carga de la cimentación.

- Estimar el asentamiento probable de una estructura.

- Determinar problemas potenciales de la cimentación como ser suelos expansivos, suelos colapsables, rellenos sanitarios, etc.

- Determinar la posición del nivel del agua.

- Predecir la presión lateral de tierra en estructuras tales como muros de retención, tablestacas y cortes apuntalados.

- Fundación

Es importante conocer el tipo de suelo a la hora de hacer la fundación de un edificio ya que esta no funciona igual en todos los tipos de suelo:

Los suelos arcillosos: son peligrosos por sus excesivos asentamientos diferidos en el tiempo. Los suelos arenosos son muy buenos para usarse como suelo de fundación.

La duración del asentamiento es responsabilidad del agua que posee el estrato y su impermeabilidad.

- Carreteras

Una adecuada clasificación permite al Ingeniero de carreteras tener una idea acerca del comportamiento que puede esperar de un suelo como cimiento del firme, a partir de propiedades como granulometría y plasticidad de un suelo para predecir su comportamiento mecánico.

La superficie de una carretera constituye solo una pequeña parte de una obra total necesaria para su construcción. Debajo de ella debe existir una base sólida preferiblemente roca, de la que depende la vida futura de la carretera, sino existe un plano de fundación bueno o resistente la superficie se agrietará y acabará cuarteándose. Los mejores planos de fundación son las rocas y las gravas apisonadas. En tales casos el resto de las carreteras (pavimento) puede construirse directamente sobre esas bases.

Los terrenos arcillosos no son recomendables como planos de fundación de carretera de carretera puesto que suelen comprimirse con la humedad bajo una carga determinada.

- Terraplenes 
Se utilizan para rellenos de predios, plataformas, caminos, bordos, pisos industriales, estacionamientos, ferrocarriles, aeropuertos, rampas de hospitales etc., son el acumulamiento de tierra o suelo de una cierta calidad, compactado.

- Elección del equipo de compactación

Según el tipo de suelo:

○ Rodillos lisos: Se utilizan en gravas y arenas mecánicamente estables.

- Rodillos neumáticos: Se usa en arenas uniformes y suelos cohesivos, humedad cercana a límite plástico.

○ Rodillos "pata de cabra": Suelos finos, humedad entre 7 a 20\% por debajo del límite plástico.

○ Rodillo vibratorio: Se utiliza especialmente en suelos granulares.

- Estanques

Para construcción de estanques es importante trabajar en suelos con un alto contenido de impermeabilidad, otorgada por las arcillas que corresponden a las partes más finas del suelo y se caracteriza por retener el agua y sustancias químicas, también es aceptable una combinación adecuada de arcillas y limos, arcillas y arenas. Un suelo apto es aquel que cuando pierde agua forma terrones bastantes fuertes y consistentes.

\section{Bibliografía}

Abreu, J. (2010). Hipótesis, método \& diseño de la investigación. México.

Angelone, S., Garibay, M. T., \&Casaux, M. C. (2006). Permeabilidad de suelos. Argentina.

Bernal, C. A. (2010). Metodología de la Investigación (3. a ed.). Colombia: Pearson Educación.

Blañón Blázquez, L. (2000). Manual de carreteras (Vol. 1). Alicante: Ortiz e Hijos, Contratista de Obras, S.A.

Buckman, H. O., \& Brady, N. C. (1966). Naturaleza y propiedades de los suelos. Barcelona: Montaner y Simón.

Camacho, C. J. (2009). Criterios Para Calificar Los Suelos Con Fines Urbanos. Recuperado 25 de febrero de 2016, a partir de http://es.slideshare.net/carlosjcamacho/criterios-para-calificar-los-suelos-confines-urbanos

Crespo, C. (2004). Mecánica de Suelos (5. ${ }^{\mathrm{a}}$ ed.). Limusa.

Das, B. M. (2001a). Fundamentos de Ingeniería Geotécnica. México: Thompson Editores.

Das, B. M. (2001b). Principios de Ingeniería de Cimentaciones (4. ${ }^{a}$ ed.). International Thompson Editores. 
Giménez Ramírez, M. del R. (2015). Capítulo Geotecnia. Recuperado a partir de http://docplayer.es/5768339-Capitulo-12-geotecnia-tabla-12-1-clasificacionde-las-rocas.html

Granados, C., Álvarez, G., \& Gale, W. (2013). Clasificación de los Suelos de la Zona Noroeste Mediante el Método SUCS (Tesis de Pregrado). San Pedro Sula, Honduras: Universidad Tecnológica Centroamericana.

Grisales Simbasica, J. (2014). Propiedades Físicas y Mecánicas de los Suelos. Colombia.

Hernández Sampieri, R., Fernández Collado, C., \& Baptista Lucio, P. (2010). Metodología de la Investigación (5. ${ }^{\mathrm{a}} \mathrm{ed}$.).

Iso 14688-1. (2001). Geotechnical investigation and testing. (1. $\left.{ }^{\mathrm{a}} \mathrm{ed}.\right)$.

Juárez Badillo, E., \& Rico Rodríguez, A. (2005). Mecánica de suelos, Tomo 1 (fundamentos de la mecánica de suelos) (3. ${ }^{\mathrm{a}}$ ed.). Limusa.

Levin, R. L., \&Rubin, D. S. (2004). Estadística Para Administración y Economía (7. a ed.). México: Pearson Educación.

Olguín Coca, F. J. M. (2011). Mecánica de Suelos 1. México.

Petróleos Mexicanos. (1975). Exploración y muestreo en suelos para proyectos de cimentaciones.

Ruiz Vázquez, M., \& González Huesca, S. (2013). Geología Aplicada a la Ingeniería Civil (5. ${ }^{\mathrm{a}}$ ed.). México: Limusa.

Walpole, R. E., Myers, R. H., Myers, S. L., \& Ye, K. (2007). Probabilidad y Estadísticas Para Ciencias E Ingenierías (8. ${ }^{a}$ ed.). México: Pearson Educación. 